Article

\title{
Synthesis of Pyridine-Dicarboxamide-Cyclohexanone Derivatives: Anticancer and $\alpha$-Glucosidase Inhibitory Activities and In Silico Study
}

\author{
Abdullah Mohammed Al-Majid ${ }^{1}$, Mohammad Shahidul Islam ${ }^{1}{ }^{(D}$, Saleh Atef ${ }^{1}$, \\ Fardous F. El-Senduny ${ }^{2}$ (D) Farid A. Badria ${ }^{3}\left(\mathbb{D}\right.$, Yaseen A. M. M. Elshaier $^{4}\left(\mathbb{D}\right.$, M. Ali $^{1}$, \\ Assem Barakat ${ }^{1,5, * \mathbb{D}}$ and A. F. M. Motiur Rahman ${ }^{6} \mathbb{D}$ \\ 1 Department of Chemistry, College of Science, King Saud University, P. O. Box 2455, \\ Riyadh 11451, Saudi Arabia; amajid@ksu.edu.sa (A.M.A.-M.); shahid.10amui@gmail.com (M.S.I.); \\ aboatef2008@gmail.com (S.A.); maly.c@ksu.edu.sa (M.A.) \\ 2 Department of Chemistry, Faculty of Science, Mansura University, Mansura 35516, Egypt; \\ biobotany@gmail.com \\ 3 Department of Pharmacognosy, Faculty of Pharmacy, Mansoura University, Mansoura 35516, Egypt; \\ faridbadria@gmail.com \\ 4 Department of Organic and Medicinal chemistry, Faculty of Pharmacy, University of Sadat City, \\ Menofia 32958, Egypt; yaseen.elshaier@fop.usc.edu.eg \\ 5 Department of Chemistry, Faculty of Science, Alexandria University, P.O. Box 426, Ibrahimia, \\ Alexandria 21321, Egypt \\ 6 Department of Pharmaceutical Chemistry, College of Pharmacy, King Saud University, \\ Riyadh 11451, Saudi Arabia; afmrahman@ksu.edu.sa \\ * Correspondence: ambarakat@ksu.edu.sa; Tel.: +966-11467-5901; Fax: +966-11467-5992
}

Academic Editor: Carmela Saturnino

Received: 8 March 2019; Accepted: 3 April 2019; Published: 4 April 2019

\begin{abstract}
An efficient and practical method for the synthesis of 2,6-diaryl-4-oxo- $N, N^{\prime}$-di(pyridin-2 -yl)cyclohexane-1,1-dicarboxamide is described in this present study, which occurs through a double Michael addition reaction between diamide and various dibenzalacetones. The reaction was carried out in dichloromethane (DCM) in the presence of 1,8-diazabicyclo[5.4.0]undec-7-ene (DBU). The anticancer activities of the synthesized compounds were evaluated in several cancer cell lines, including MCF-7, MDA-MB-231, SAS, PC-3, HCT-116, HuH-7 and HepG2 cells. From these experiments, we determined that MDA-MB-231 was the most sensitive cancer cell line to the compounds $\mathbf{3 c}, \mathbf{3 e}, \mathbf{3 d}, \mathbf{3} \mathbf{j}$ and $\mathbf{3 l}$, which exhibited variable anticancer activities $\left(\mathbf{3 1}\left[\mathrm{IC}_{50}=5 \pm 0.25 \mu \mathrm{M}\right]\right.$ $\left.>3 \mathrm{e}\left[\mathrm{IC}_{50}=5 \pm 0.5 \mu \mathrm{M}\right]>3 \mathbf{c}\left[\mathrm{IC}_{50}=7 \pm 1.12 \mu \mathrm{M}\right]>3 \mathbf{d}\left[\mathrm{IC}_{50}=18 \pm 0.87 \mu \mathrm{M}\right]>3 \mathbf{j}\left[\mathrm{IC}_{50}=45 \pm 3 \mu \mathrm{M}\right]\right)$. Of these, 31 (substituted $p$-trifluoromethylphenyl and chloropyridine) showed good potency $\left(\mathrm{IC}_{50}=6 \pm 0.78 \mu \mathrm{M}\right)$ against HCT-116 colorectal cancer cells and exhibited high toxicity against HuH-7 liver cancer cells $\left(\mathrm{IC}_{50}=4.5 \pm 0.3 \mu \mathrm{M}\right)$. These values were three times higher than the values reported for cisplatin ( $\mathrm{IC}_{50}$ of $8 \pm 0.76$ and $14.7 \pm 0.5 \mu \mathrm{M}$ against HCT-116 and HuH-7 cells, respectively). The highest $\alpha$-glucosidase inhibitory activity was detected for the $\mathbf{3} \mathbf{d}, \mathbf{3 i}$ and $\mathbf{3} \mathbf{j}$ compounds. The details of the binding mode of the active compounds were clarified by molecular docking studies.
\end{abstract}

Keywords: malonamide; Michael addition reaction; cytotoxicity; cancer; $\alpha$-glucosidase; docking

\section{Introduction}

Several malonamide-based anticancer agents with promising cytotoxic activities have been identified from natural and synthetic sources [1,2]. For instance, golvatinib (E-7050) is a clinical agent 
with dual inhibitory activity against c-Met and vascular endothelial growth factor receptor-2 (VEGFR-2) tyrosine kinases and is known to exhibit high antineoplastic potential [3] (Figure 1). BMS-777607, one of the malonamide-based molecules with Met inhibition activity, has entered phase II clinical trials [4-7]. Chu et al. provided a malonamide-based small molecule I, which is thought to be effective as a selective $\kappa$ optical receptor agonist [8]. Our research team recently developed several malonamide motifs as $\alpha$-glucosidase inhibitory agents $[9,10]$, which have moderate cytotoxicity against HeLa, H460, MCF-7 and 3T3 cell lines [9].

Functionalized cyclohexanones that utilizes stereogenic centers as valuable building blocks are known to be present at the core of several natural products and drug candidates. Functionalized cyclohexanones are embedded in the antidepressant and dissociative anesthetic drugs Ketanest ${ }^{\circledR}$ S [11] and Vasoxyl ${ }^{\circledR}$ methoxamine (for the treatment of hypotension) [12]. These molecules possess antibacterial [13], anticonvulsant [14], antifungal and anticancer [15] properties. In general, cyclohexanone is a common scaffold in various bioactive heterocycles of medicinal interests, particularly those used for the treatment of asthma and central nervous system (CNS)- and chronic obstructive pulmonary diseases (COPD)-related diseases, due to its inhibitory activity against phosphodiesterase 4 (PDE4) $[16,17]$.

As a continuation of our search for malonamide-based potent anticancer agents, in this present study, we demonstrate the preparation of a new library of malonamide-based compounds $(3 \mathbf{a}-\mathbf{m})$ through the incorporation of important scaffolds, namely cyclohexanone and dicarboximide derivatives, in a single molecule and highlight their anticancer and $\alpha$-glucosidase inhibitory activities.

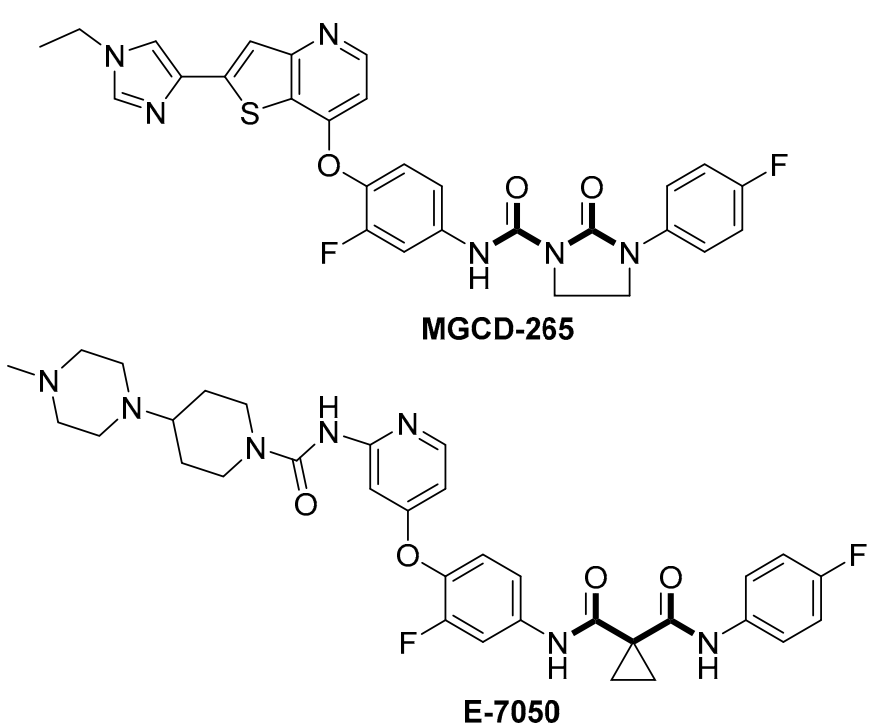<smiles>CCOc1ccn(-c2ccc(F)cc2)c(=O)c1C(=O)Nc1ccc(Oc2ccnc(N)c2Cl)c(F)c1</smiles>

Figure 1. Structures of some biologically active $N, N^{\prime}$-malonamide derivatives.

\section{Results}

\subsection{Synthesis of $\mathbf{3 a}-\mathbf{m}$}

Anticancer compounds incorporating 2,6-diaryl-4-oxo- $N, N^{\prime}$-di(pyridin-2-yl)cyclohexane-1, 1-dicarboxamide $3 \mathbf{a}-\mathbf{m}$ via a double Michael addition reaction were prepared according to the previously described method [8,9]. The reaction was carried out by mixing diamide $\mathbf{1 a}, \mathbf{b}$ that was carrying an active methylene group with dienone $\mathbf{2 a - m}$ (Scheme 1, Table 1) in dichloromethane $(\mathrm{DCM})$ at room temperature $\left(24^{\circ} \mathrm{C}\right)$ for $2-3 \mathrm{~h}$. The process was carried out in the presence of DBU (1,8-Diazabicyclo[5.4.0]undec-7-ene) to obtain the final compound 3a-m at an acceptable yield (33-89\%). The chemical structures of the Michael-adducts were deduced with infrared (IR) spectroscopy, mass spectrometry (MS), ${ }^{1} \mathrm{H}$-nuclear magnetic resonance (NMR), ${ }^{13} \mathrm{C}-\mathrm{NMR}$ and elemental analysis (CHN). 


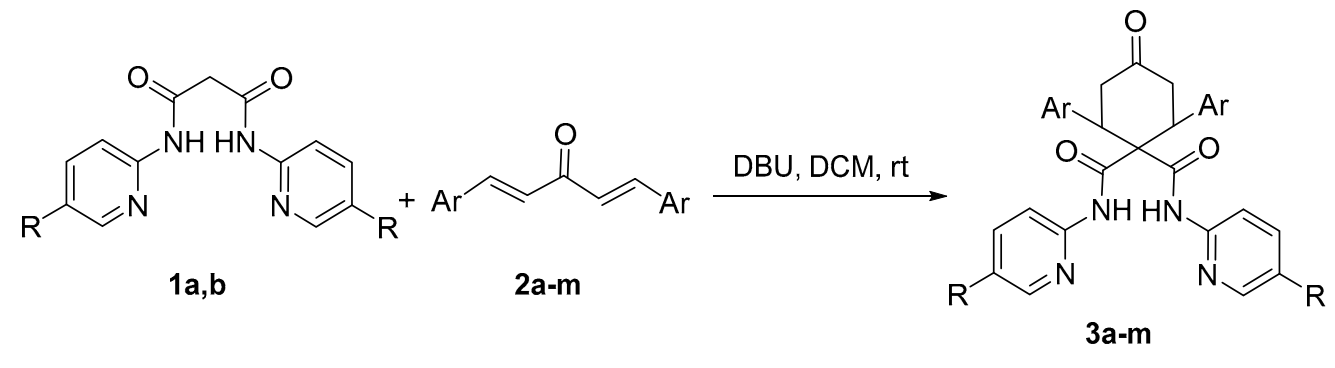

Scheme 1. The synthesis of the target compound 3a-m.

Table 1. Procedure for the synthesis of the target compound 3a-m.

\begin{tabular}{cccccc}
\hline$\#$ & $\mathbf{R}$ & $\mathbf{2 a - m}$ & $\mathbf{A r}$ & Product 3a-m & Yield, $\mathbf{0}$ \\
\hline 1 & $\mathrm{H}$ & $\mathbf{2 a}$ & $\mathrm{C}_{6} \mathrm{H}_{5}$ & $\mathbf{3 a}$ & 89 \\
\hline 2 & $\mathrm{Cl}$ & $\mathbf{2 b}$ & $p-\mathrm{CH}_{3} \mathrm{C}_{6} \mathrm{H}_{4}$ & $\mathbf{3 b}$ & 33 \\
\hline 3 & $\mathrm{H}$ & $\mathbf{2 c}$ & $p-\mathrm{ClC}_{6} \mathrm{H}_{4}$ & $\mathbf{3 c}$ & 64 \\
\hline 4 & $\mathrm{H}$ & $\mathbf{2 d}$ & $2,4-\mathrm{Cl}_{2} \mathrm{C}_{6} \mathrm{H}_{3}$ & $\mathbf{3 d}$ & 60 \\
\hline 5 & $\mathrm{H}$ & $\mathbf{2 e}$ & $p-\mathrm{BrC}_{6} \mathrm{H}_{4}$ & $\mathbf{3 e}$ & 56 \\
\hline 6 & $\mathrm{Cl}$ & $\mathbf{2 f}$ & $m-\mathrm{NO}_{2} \mathrm{C}_{6} \mathrm{H}_{4}$ & $\mathbf{3 f}$ & 62 \\
\hline 7 & $\mathrm{Cl}$ & $\mathbf{2 g}$ & $p-\mathrm{MeOC}_{6} \mathrm{H}_{4}$ & $\mathbf{3 g}$ & 72 \\
\hline 8 & $\mathrm{Cl}$ & $\mathbf{2 h}$ & $\beta-\mathrm{Naphthalene}$ & $\mathbf{3 h}$ & 58 \\
\hline 9 & $\mathrm{Cl}$ & $\mathbf{2 i}$ & $2-\mathrm{Thiophene}$ & $\mathbf{3 i}$ & 60 \\
\hline 10 & $\mathrm{Cl}$ & $\mathbf{2 j}$ & $2-\mathrm{Furan}_{2}$ & $\mathbf{3 j}$ & 52 \\
\hline 11 & $\mathrm{Cl}$ & $\mathbf{2 k}$ & $m-\mathrm{BrC}_{6} \mathrm{H}_{4}$ & $\mathbf{3 k}$ & 44 \\
\hline 12 & $\mathrm{Cl}$ & $\mathbf{2 1}$ & $p-\mathrm{CF}_{3} \mathrm{C}_{6} \mathrm{H}_{4}$ & $\mathbf{3 1}$ & 37 \\
\hline 13 & $\mathrm{H}$ & $\mathbf{2 m}$ & $p-\mathrm{FC}_{6} \mathrm{H}_{4}$ & $\mathbf{3 m}$ & 85 \\
\hline
\end{tabular}

\subsection{Biological Activities}

\subsubsection{Anticancer Activity}

To evaluate the anticancer activity of the 13 newly synthesized compounds, we screened their activities at a concentration of $50 \mu \mathrm{M}$ against seven cancer cell lines, including breast cancer (positive [MCF-7] and negative [MDA-MB-231] for estrogen receptor expression), tongue carcinoma (SAS), prostate cancer (PC-3), colorectal cancer (HCT-116) and liver cancer (HuH-7 and HepG2) cell lines. The results revealed that only five compounds (3c, 3d, 3e, 3k and 31) showed different levels of anticancer activities (Table 2). The 3-(4,5-dimethylthiazol-2-yl)-2,5-diphenyltetrazolium bromide (MTT) assay was performed to determine the concentration of the active compounds needed to kill $50 \%$ of cells. The results revealed that the compound 3c (substituted $p$-chlorophenyl) killed 50\% of ER-negative breast cancer cells $\left(\mathrm{IC}_{50}=7 \pm 1.12 \mu \mathrm{M}\right)$ and HepG2 $\left(\mathrm{IC}_{50}=8 \pm 0.89 \mu \mathrm{M}\right)$ at concentrations lower than that of the common chemotherapeutic drug cisplatin $\left(\mathrm{IC}_{50}=15 \pm 0.71\right.$ and $10 \pm 0.65 \mu \mathrm{M}$ against ER-negative breast cancer cells and HepG2, respectively). Furthermore, the anticancer activities of the compounds $3 \mathbf{e}$ (substituted $p$-bromophenyl, $\mathrm{IC}_{50}=5 \pm 0.5 \mu \mathrm{M}$ ) and 31 (substituted $p$-trifluoromethylphenyl and chloropyridine, $\mathrm{IC}_{50}=5 \pm 0.25 \mu \mathrm{M}$ ) were stronger than the activity of cisplatin $\left(\mathrm{IC}_{50}=15 \pm 0.71 \mu \mathrm{M}\right)$ against MDA-MB-231 cells. The compounds $3 \mathbf{c}, \mathbf{3 e}$ and 31 also exhibited moderate anticancer activities against the ER-positive MCF-7 cell lines $\left(\mathrm{IC}_{50}=10 \pm 0.62,12 \pm 0.54\right.$ and $18 \pm 1.71 \mu \mathrm{M}$, respectively). Only two compounds (3c and 31) exhibited moderate anticancer activities against the tongue carcinoma cell line (SAS, $\mathrm{IC}_{50}=15 \pm 1.3$ and $9 \pm 0.38 \mu \mathrm{M}$, respectively). Of these molecules, the compound 31 (substituted $p$-trifluoromethylphenyl and chloropyridine) showed good potency $\left(\mathrm{IC}_{50}=6 \pm 0.78 \mu \mathrm{M}\right)$ against HCT-116 colorectal cancer cells and exhibited high efficacy 
against $\mathrm{HuH}-7$ liver cancer cells $\left(\mathrm{IC}_{50}=4.5 \pm 0.3 \mu \mathrm{M}\right)$. These values were three times higher than the values reported for cisplatin $\left(\mathrm{IC}_{50}\right.$ of $8 \pm 0.76$ and $14.7 \pm 0.5 \mu \mathrm{M}$ against HCT-116 and HuH-7 cells, respectively) (Table 2).

Table 2. The cytotoxic activities of the test compounds against seven cancer cell lines representing five different types of cancers (breast, tongue, prostate, colon and liver). Cell viability was evaluated with the MTT assay and the $\mathrm{IC}_{50}(\mu \mathrm{M})$ value was calculated. The values are represented as the mean \pm standard deviation from three independent experiments. NA indicates that the compounds were not active during the initial screening of their anticancer activities using crystal violet assay.

\begin{tabular}{|c|c|c|c|c|c|c|c|}
\hline \multirow{2}{*}{ Compounds ${ }^{a, b}$} & \multicolumn{2}{|c|}{ Breast } & \multirow{2}{*}{$\begin{array}{l}\text { Oral } \\
\text { SAS }\end{array}$} & \multirow{2}{*}{$\begin{array}{c}\text { Prostate } \\
\text { PC-3 }\end{array}$} & \multirow{2}{*}{$\begin{array}{c}\text { Colon } \\
\text { HCT-116 }\end{array}$} & \multicolumn{2}{|c|}{ Liver } \\
\hline & MCF-7 & MDA-MB-231 & & & & HuH-7 & HepG2 \\
\hline $3 a$ & $\mathrm{NA}^{\mathrm{c}}$ & NA & NA & NA & NA & NA & NA \\
\hline $3 b$ & NA & NA & NA & NA & NA & NA & NA \\
\hline $3 c$ & $10 \pm 0.62$ & $7 \pm 1.12$ & $15 \pm 1.3$ & $25 \pm 1.42$ & NA & NA & $8 \pm 0.89$ \\
\hline $3 d$ & $>50 \pm 1.17$ & $18 \pm 0.87$ & NA & NA & NA & NA & $>50$ \\
\hline $3 e$ & $12 \pm 0.54$ & $5 \pm 0.5$ & NA & NA & NA & NA & $8 \pm 0.96$ \\
\hline $3 f$ & NA & NA & NA & NA & NA & NA & NA \\
\hline $3 g$ & NA & NA & NA & NA & NA & NA & NA \\
\hline $3 \mathrm{~h}$ & NA & NA & NA & NA & NA & NA & NA \\
\hline $3 \mathbf{i}$ & NA & NA & NA & NA & NA & NA & NA \\
\hline $3 \mathbf{j}$ & $50 \pm 0.78$ & $45 \pm 3$ & NA & NA & NA & NA & $>50 \pm 1.08$ \\
\hline $3 k$ & NA & NA & NA & NA & NA & NA & NA \\
\hline 31 & $18 \pm 1.71$ & $5 \pm 0.25$ & $9 \pm 0.38$ & $>50 \pm 2$ & $6 \pm 0.78$ & $4.5 \pm 0.3$ & $25 \pm 0.38$ \\
\hline $3 m$ & NA & NA & NA & NA & NA & NA & NA \\
\hline Cisplatin & $9 \pm 2.43$ & $15 \pm 0.71$ & $4.5 \pm 0.34$ & $12 \pm 1.25$ & $8 \pm 0.76$ & $14.7 \pm 0.5$ & $10 \pm 0.65$ \\
\hline
\end{tabular}

${ }^{a}$ All test compounds showed a value of $\mathrm{IC}_{50}>100 \mu \mathrm{M}$ against all seven cell lines (very high safety margin); ${ }^{\mathrm{b}}$ In comparison with cisplatin $\mathrm{IC}_{50}(\mu \mathrm{M})$ value, which was very marginal and in the range of $15-20 \mu \mathrm{M}$ for MCF-7, MDA-MB-231, SAS, PC-3, HCT-11, HuH-7 and HepG2; ${ }^{\mathrm{c}} \mathrm{NA}$, no or negligible activity.

\subsection{2. $\alpha$-Glucosidase Inhibitory Activity}

The synthesized compounds were screened for their ability to inhibit $\alpha$-glucosidase activity and the results are summarized in Table 3. Among all the compounds, $\mathbf{3} \mathbf{d}, \mathbf{3 i}$ and $\mathbf{3} \mathbf{j}$ exhibited excellent $\alpha$-glucosidase inhibitory activities while the rest of the compounds were inactive.

However, the compound $\mathbf{3} \mathbf{j}$ (substituted furan and chloropyridine moieties) showed the highest $\alpha$-glucosidase inhibitory activity with an $\mathrm{IC}_{50}$ value of $124.24 \pm 0.16 \mu \mathrm{mol} / \mathrm{L}$, followed by the compounds $3 \mathbf{d}$ (substituted 2,4-dichlorobenzene and pyridine moieties; $\mathrm{IC}_{50}$ of $148.18 \pm 3.02 \mu \mathrm{mol} / \mathrm{L}$ ) and $3 \mathbf{i}$ (substituted thiophene and chloropyridine moieties; $\mathrm{IC}_{50}$ of $418.21 \pm 1.02 \mu \mathrm{mol} / \mathrm{L}$ ). Acarbose was used as a standard control $\left(\mathrm{IC}_{50}=32.71 \pm 1.17 \mu \mathrm{mol} / \mathrm{L}\right)$.

Table 3. Results of $\alpha$-glucosidase inhibitory activity.

\begin{tabular}{cc}
\hline Compounds & IC $_{\mathbf{5 0}}( \pm$ SEM $\boldsymbol{\mu m o l} / \mathrm{L})$ \\
\hline $\mathbf{3 d}$ & $148.18 \pm 3.02$ \\
\hline $\mathbf{3 i}$ & $418.21 \pm 1.02$ \\
\hline $\mathbf{3} \mathbf{j}$ & $124.24 \pm 0.16$ \\
\hline Acarbose & $32.71 \pm 1.17$ \\
\hline
\end{tabular}




\subsection{Molecular Docking Study}

As evident from the data represented in Table 1, the synthesized compounds exhibited diversity in their anticancer activities and only compounds $31,3 \mathrm{c}$ and $3 \mathbf{e}$ exerted strong anticancer activities. We subsequently investigated the protein that interacts with these three compounds in a unique binding mode and exhibits strong binding interactions in a manner different from those with the inactive analogues. Docking procedures were performed in the presence of different proteins, including tyrosine kinase (ID: 3F82 [6], mammalian target of rapamycin (mTOR; ID: 4JSV) [18], epidermal growth factor receptor (EGFR; ID: 1M17) [19] and extracellular signal-regulated kinase (ERK; ID: 2OJG) [20] and 2OJJ [20-23].

We found that the active compounds docked well with EGFR and showed a specific strong interaction pattern. The compound 31 formed HB (acceptor) with the oxygen of amidic carbonyl of the amino acid residue Gly 772 AA, (Figure 2A). This amino acid interacts with the standard erlotinib in a non-HB manner [19]. Both the compounds $3 \mathbf{e}$ and $3 \mathbf{c}$ showed similar behavior in terms of binding mode and docking pose with the receptor through hydrophobic-hydrophobic interactions (Figure 2B). The inactive analogs, such as the compound $3 \mathrm{~m}$, showed a different binding interaction in comparison to $3 \mathbf{c}$ and $3 \mathbf{e}$.

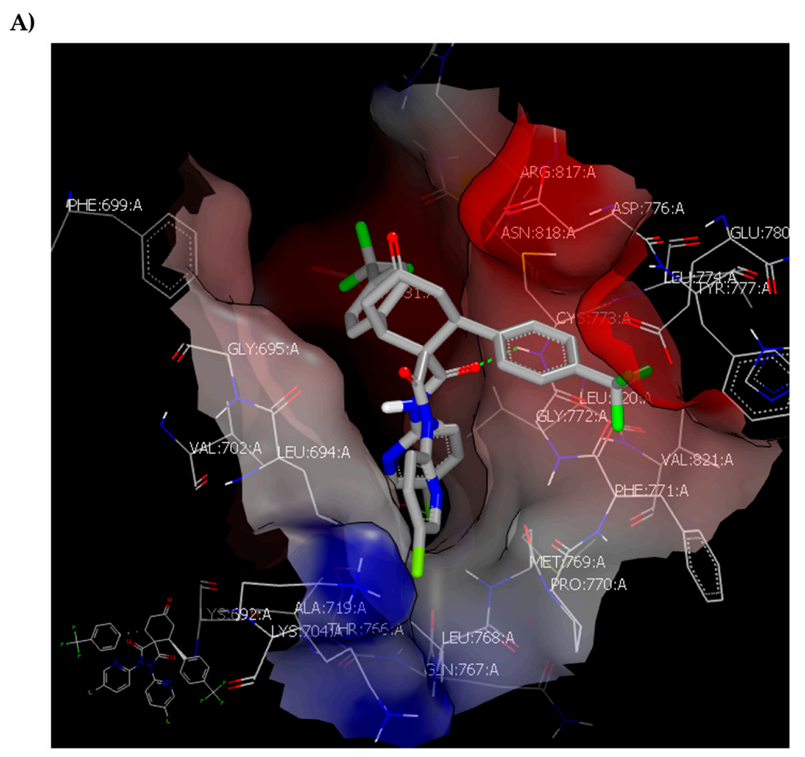

B)

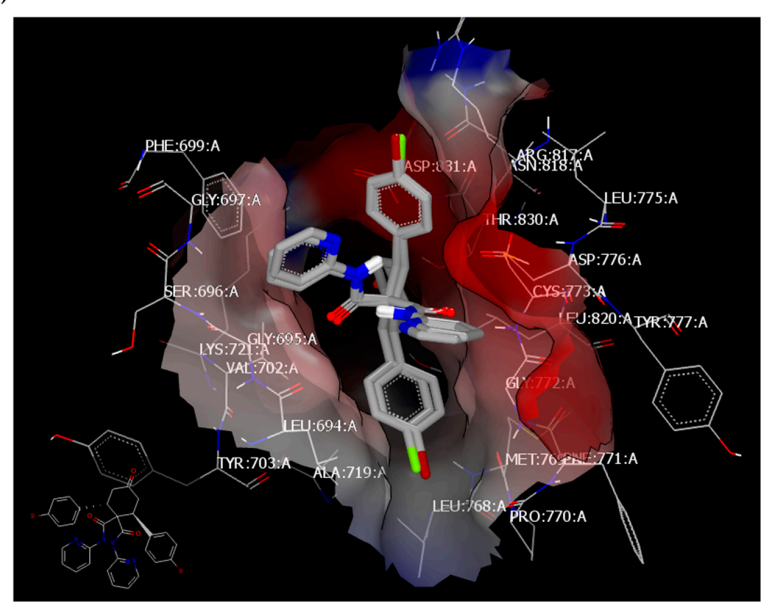

Figure 2. (A) Snapshot of 31 in ID:1M7 showing the HB interaction with Gly 772; (B) Snapshot of 3c and 3e (ID:1M17) overlaid on each other to show the hydrophobic-hydrophobic interactions. 


\subsection{Structure-Activity Relationship (SAR)}

The reason underlying the potent activity of only those three compounds was further investigated. The analysis showed that there is a similarity in the three-dimensional shape and electrostatic potential of those three compounds [24]. Shape similarity (3D similarity) is considered to be a fundamental descriptor for computational drug discovery and is an important characteristic to correctly model and accurately understand the protein-ligand interaction. The shape provides information on neighborhood behavior and the high similarity in shape is reflective of the consistent biological properties [25].

The final compounds contained four aromatic rings as the substituents of the cyclohexanone ring $(3,4,4,5)$, which indicates its highly lipophilic nature that may facilitate the efflux of drugs outside the cells and subsequently decrease the activity.

Based on the docking results for all final compounds, it was found that the pyridine carboxamides and the para-substituent in the phenyl ring linked to the cyclohexanone ring determine the geometry of each compound (3D structure) and reflect the orientation of each scaffold in the side of the receptor clefts. The presence of the dipyridine carboxamide skeleton is essential for the activity of the compound. The substitution of pyridine ring is not important, while the para-substitution with an electron-withdrawing group (except fluorine) on the aromatic moiety is essential.

\section{Materials and Methods}

\subsection{Experimental}

General procedure (GP): Dienones $\mathbf{2} \mathbf{a}-\mathbf{m}(0.25 \mathrm{mmol})$ and diamide $\mathbf{1 a}$ or $\mathbf{1 b}(74 \mathrm{mg}, 0.25 \mathrm{mmol})$ were dissolved in $10 \mathrm{~mL}$ of dry $\mathrm{CH}_{2} \mathrm{Cl}_{2}$ in a $25 \mathrm{~mL}$ round bottom flask. DBU (3 eq, $114 \mathrm{mg}, 0.75 \mathrm{mmol}$ ) was added to the reaction, which was subsequently stirred for 2-3 h. After the reaction was completed as determined by TLC, the crude material was subjected to column chromatography using ethyl acetate/ $n$-hexane (2:3) to give the desired compounds $3 \mathbf{a}-\mathbf{m}$.

4-Oxo-2,6-diphenyl- $N, N^{\prime}$-di(pyridin-2-yl)cyclohexane-1,1-dicarboxamide $\quad$ (3a). Yield $120 \quad \mathrm{mg}$ (0.22 mmol, 89\%); m.p. 248-249 ${ }^{\circ} \mathrm{C} ;{ }^{1} \mathrm{H}-\mathrm{NMR}\left(\mathrm{DMSO}-d_{6}, 400 \mathrm{MHz}\right) \delta: 2.35$ and 2.37 (dd, $2 \mathrm{H}, \mathrm{J}=4.4 \mathrm{~Hz}$ and $\left.11.6 \mathrm{~Hz}, \mathrm{CH}_{2}\right), 2.69(\mathrm{t}, 2 \mathrm{H}, J=12.0 \mathrm{~Hz}, \mathrm{CH}), 3.99$ and $4.01\left(\mathrm{dd}, 2 \mathrm{H}, J=4.8 \mathrm{~Hz}\right.$ and $10.4 \mathrm{~Hz}_{2} \mathrm{CH}_{2}$ ), $6.87(\mathrm{t}, 1 \mathrm{H}, J=6.8 \mathrm{~Hz}, \mathrm{Ar}-\mathrm{H}), 6.94(\mathrm{~s}, 1 \mathrm{H}, \mathrm{NH}), 7.06(\mathrm{t}, 2 \mathrm{H}, J=7.2 \mathrm{~Hz}, \mathrm{Ar}-\mathrm{H}), 7.17(\mathrm{t}, 4 \mathrm{H}, J=8.0 \mathrm{~Hz}$, Ar-H); 7.36 (d, 4H, J = 7.6 Hz, Ar-H), 7.47-7.58 (m, 3H, Ar-H), 7.76 (d, 1H, J = 8.4 Hz, Ar-H), 7.93 (d, $1 \mathrm{H}, J=4.4 \mathrm{~Hz}, \mathrm{Ar}-\mathrm{H}), 8.02(\mathrm{dt}, 1 \mathrm{H}, J=2.0 \mathrm{~Hz}$ and $5.6 \mathrm{~Hz}, \mathrm{Ar}-\mathrm{H}), 8.70$ and $8.71(\mathrm{dd}, 1 \mathrm{H}, J=1.2 \mathrm{~Hz}$ and $4.8 \mathrm{~Hz}, \mathrm{Ar}-\mathrm{H}), 11.11$ (s, 1H, NH); ${ }^{13} \mathrm{C}-\mathrm{NMR}$ (DMSO-d $\left.6,100 \mathrm{MHz}\right) \delta: 42.9,46.1,58.7,87.3,113.5$, $119.9,123.8,125.3,127.4,128.7,128.6,138.3,138.6,142.2,148.2,149.8,150.4,150.6,168.1,170.4 ; \mathrm{IR}(\mathrm{KBr}$, $\left.\mathrm{cm}^{-1}\right) v_{\max }=3434,3028,1688,1651,1574,1532,1467,1403 ., 1296,1161,757,702,555$; [Anal. Calcd. for $\mathrm{C}_{30} \mathrm{H}_{26} \mathrm{~N}_{4} \mathrm{O}_{3}$ : C, 73.45; H, 5.34; N, 11.42; Found: C, 73.57; H, 5.46; N, 11.33]; LC/MS (ESI, $\left.m / z\right)$ : [M+], found $490.20, \mathrm{C}_{30} \mathrm{H}_{26} \mathrm{~N}_{4} \mathrm{O}_{3}$ for 490.20 .

$N, N^{\prime}$-bis(5-Chloropyridin-2-yl)-4-oxo-2,6-di-p-tolylcyclohexane-1,1-dicarboxamide (3b). Yield $48 \mathrm{mg}$ (0.09 mmol, 33\%); m.p. 145-146 ${ }^{\circ} \mathrm{C} ;{ }^{1} \mathrm{H}-\mathrm{NMR}$ (DMSO- $\left.d_{6}, 400 \mathrm{MHz}\right) \delta: 2.10$ (s, 6H, $\left.\mathrm{CH}_{3}\right), 2.29-2.33$ (m, $\left.2 \mathrm{H}, \mathrm{CH}_{2}\right), 2.66(\mathrm{t}, 2 \mathrm{H}, \mathrm{J}=11.4 \mathrm{~Hz}, \mathrm{CH}), 3.93$ and $3.95\left(\mathrm{dd}, 2 \mathrm{H}, \mathrm{J}=4.8 \mathrm{~Hz}\right.$ and $\left.11.4 \mathrm{~Hz}, \mathrm{CH}_{2}\right), 6.96(\mathrm{~d}, 4 \mathrm{H}$, $J=8.0 \mathrm{~Hz}, \mathrm{Ar}-\mathrm{H}), 7.03(\mathrm{~s}, 1 \mathrm{H}, \mathrm{NH}), 7.18(\mathrm{~d}, 4 \mathrm{H}, J=8.0 \mathrm{~Hz}, \mathrm{Ar}-\mathrm{H}), 7.55(\mathrm{~d}, 1 \mathrm{H}, J=8.4 \mathrm{~Hz}, \mathrm{Ar}-\mathrm{H}), 7.70$ and $7.72(\mathrm{dd}, 1 \mathrm{H}, J=2.8 \mathrm{~Hz}$ and $9.2 \mathrm{~Hz}, \mathrm{Ar}-\mathrm{H}), 7.83(\mathrm{~d}, 1 \mathrm{H}, J=8.4 \mathrm{~Hz}, \mathrm{Ar}-\mathrm{H}), 8.01(\mathrm{~d}, 1 \mathrm{H}, J=2.0 \mathrm{~Hz}$, Ar-H), 8.14 and $8.16(\mathrm{dd}, 1 \mathrm{H}, J=2.8 \mathrm{~Hz}$ and $8.0 \mathrm{~Hz}, \mathrm{Ar}-\mathrm{H}), 8.76(\mathrm{~d}, 1 \mathrm{H}, J=2.4 \mathrm{~Hz}, \mathrm{Ar}-\mathrm{H}), 11.16(\mathrm{~s}, 1 \mathrm{H}$, $\mathrm{NH}) ;{ }^{13} \mathrm{C}-\mathrm{NMR}\left(\mathrm{DMSO}-d_{6}, 100 \mathrm{MHz}\right) \delta: 20.9,42.8,45.7,58.9,87.6,114.5,125.6,126.6,128.5,129.3,130.9$, $136.4,138.2,138.4,139.0,146.6,148.3,148.2,149.2,168.4,170.7 ; \mathrm{IR}\left(\mathrm{KBr}, \mathrm{cm}^{-1}\right) v_{\max }=\operatorname{IR}\left(\mathrm{KBr}_{,} \mathrm{cm}^{-1}\right)$ $v \max =3231,3088,1737,1691,1656,1563,1468,1375,1281,1244,1175,1112,1014,828,807$, 682; [Anal. Calcd. for $\mathrm{C}_{32} \mathrm{H}_{28} \mathrm{Cl}_{2} \mathrm{~N}_{4} \mathrm{O}_{3}$ : C, 65.42; $\mathrm{H}, 4.80$; N, 9.54; Found: C, 65.31; H, 4.93; N, 9.67; LC/MS (ESI, $m / z):[\mathrm{M}+]$, found 586.10; $\mathrm{C}_{32} \mathrm{H}_{28} \mathrm{Cl}_{2} \mathrm{~N}_{4} \mathrm{O}_{3}$ for 586.15 . 
2,6-bis(4-Chlorophenyl)-4-oxo- $N, N^{\prime}$-di(pyridin-2-yl)cyclohexane-1,1-dicarboxamide (3c). Yield $90 \mathrm{mg}$ (0.16 mmol, 64\%); m.p. $231-232{ }^{\circ} \mathrm{C} ;{ }^{1} \mathrm{H}-\mathrm{NMR}$ (DMSO- $\left.d_{6}, 400 \mathrm{MHz}\right) \delta: 2.30$ and $2.33(\mathrm{dd}, 2 \mathrm{H}, J=5.6 \mathrm{~Hz}$ and $\left.12.0 \mathrm{~Hz}, \mathrm{CH}_{2}\right), 2.69(\mathrm{t}, 2 \mathrm{H}, J=12.4 \mathrm{~Hz}, \mathrm{CH}), 4.00$ and $4.02\left(\mathrm{dd}, 2 \mathrm{H}, J=4.8 \mathrm{~Hz}\right.$ and $\left.10.4 \mathrm{~Hz}, \mathrm{CH}_{2}\right)$, $6.87(\mathrm{t}, 1 \mathrm{H}, J=4.8 \mathrm{~Hz}, \mathrm{Ar}-\mathrm{H}), 7.01(\mathrm{~s}, 1 \mathrm{H}, \mathrm{NH}), 7.23(\mathrm{~d}, 4 \mathrm{H}, J=8.4 \mathrm{~Hz}, \mathrm{Ar}-\mathrm{H}) ; 7.37(\mathrm{~d}, 4 \mathrm{H}, J=8.4 \mathrm{~Hz}$, Ar-H), 7.48-7.51 (m, 1H, Ar-H), $7.54(\mathrm{~d}, 1 \mathrm{H}, J=8.0 \mathrm{~Hz}, \mathrm{Ar}-\mathrm{H}), 7.60(\mathrm{t}, 1 \mathrm{H}, J=7.2 \mathrm{~Hz}, \mathrm{Ar}-\mathrm{H}), 7.76(\mathrm{~d}$, $1 \mathrm{H}, J=8.8 \mathrm{~Hz}, \mathrm{Ar}-\mathrm{H}), 7.98-8.03$ (m, 2H, Ar-H), 8.69-8.70 (m, 1H, Ar-H), 11.11 (s, 1H, NH); ${ }^{13} \mathrm{C}-\mathrm{NMR}$ (DMSO- $\left.d_{6}, 100 \mathrm{MHz}\right) \delta: 42.7,45.3,58.5,87.2,113.5,120.2,124.0,125.5,128.7,130.6,132.1,138.5,138.7$, 141.0, 148.4, 149.8, 150.2, 150.4, 167.9, 170.2; IR $\left(\mathrm{KBr}, \mathrm{cm}^{-1}\right) v_{\max }=3434,3075,1695,1651,1590,1577$, 1531, 1469, 1433, 1327, 1295, 1161, 1110, 1053, 991, 774, 555; [Anal. Calcd. for $\mathrm{C}_{30} \mathrm{H}_{24} \mathrm{Cl}_{2} \mathrm{~N}_{4} \mathrm{O}_{3}$ : C, 64.41; H, 4.32; N, 10.01; Found: C, 64.12; H, 4.53; N, 10.15]; LC/MS (ESI, $m / z)$ : [M+], found 558.10, $\mathrm{C}_{30} \mathrm{H}_{24} \mathrm{Cl}_{2} \mathrm{~N}_{4} \mathrm{O}_{3}$ for 558.12 .

2,6-bis(2,4-Dichlorophenyl)-4-oxo- $N, N^{\prime}$-di(pyridin-2-yl)cyclohexane-1,1-dicarboxamide (3d). Yield $94 \mathrm{mg}$ (0.15 mmol, 60\%); m.p. 210-211 ${ }^{\circ} \mathrm{C} ;{ }^{1} \mathrm{H}-\mathrm{NMR}$ (DMSO- $\left.d_{6}, 400 \mathrm{MHz}\right) \delta: 2.03$ and $2.05(\mathrm{dd}, 2 \mathrm{H}$, $J=5.2 \mathrm{~Hz}$ and $\left.11.6 \mathrm{~Hz}, \mathrm{CH}_{2}\right), 2.89(\mathrm{t}, 2 \mathrm{H}, J=11.6 \mathrm{~Hz}, \mathrm{CH}), 4.53$ and $4.55(\mathrm{dd}, 2 \mathrm{H}, J=6.0 \mathrm{~Hz}$ and $11.2 \mathrm{~Hz}$, $\left.\mathrm{CH}_{2}\right), 6.94-6.97(\mathrm{~m}, 1 \mathrm{H}, \mathrm{Ar}-\mathrm{H}), 7.08(\mathrm{~s}, 1 \mathrm{H}, \mathrm{NH}), 7.37(\mathrm{~d}, 1 \mathrm{H}, J=7.6 \mathrm{~Hz}, \mathrm{Ar}-\mathrm{H}) ; 7.39(\mathrm{~d}, 1 \mathrm{H}, J=2.0 \mathrm{~Hz}$, Ar-H), $7.41(\mathrm{~d}, 1 \mathrm{H}, J=2.0 \mathrm{~Hz}, \mathrm{Ar}-\mathrm{H}), 7.45(\mathrm{~d}, 2 \mathrm{H}, J=2.4 \mathrm{~Hz}, \mathrm{Ar}-\mathrm{H}), 7.47-7.50(\mathrm{~m}, 1 \mathrm{H}, \mathrm{Ar}-\mathrm{H}), 7.58-7.62$ $(\mathrm{m}, 1 \mathrm{H}, \mathrm{Ar}-\mathrm{H}), 7.68(\mathrm{t}, 3 \mathrm{H}, J=8.4 \mathrm{~Hz}, \mathrm{Ar}-\mathrm{H}), 7.97(\mathrm{dt}, 1 \mathrm{H}, J=2.0 \mathrm{~Hz}$ and 7.6, Ar-H), 8.05-8.06 $(\mathrm{m}, 1 \mathrm{H}$, Ar-H), 8.65-8.67 (m, $1 \mathrm{H}, \mathrm{Ar}-\mathrm{H}), 11.00$ (s, $1 \mathrm{H}, \mathrm{NH}) ;{ }^{13} \mathrm{C}-\mathrm{NMR}$ (DMSO- $\left.d_{6}, 100 \mathrm{MHz}\right) \delta: 41.9,42.8,55.9$, 86.9, 113.5, 120.3, 124.2, 125.4, 128.5, 129.3, 132.6, 134.6, 138.6, 138.8, 139.7, 148.4, 149.8, 149.9, 150.5, 166.9, 171.1; IR $\left(\mathrm{KBr}, \mathrm{cm}^{-1}\right) v_{\max }=3435,3076,1694,1650,1589,1573,1530,1467,1435,1329,1296,1163$, 1111, 1050, 995, 820, 775, 569; [Anal. Calcd. for $\mathrm{C}_{30} \mathrm{H}_{22} \mathrm{Cl}_{4} \mathrm{~N}_{4} \mathrm{O}_{3}$ : C, 57.35; $\mathrm{H}, 3.53 ; \mathrm{N}, 8.92$; Found: $\mathrm{C}$, 57.54; $\mathrm{H}, 3.67 ; \mathrm{N}$, 9.13]; LC/MS (ESI, $m / z$ ): [M+], found 626.10, $\mathrm{C}_{30} \mathrm{H}_{22} \mathrm{Cl}_{4} \mathrm{~N}_{4} \mathrm{O}_{3}$ for 626.04.

2,6-bis(4-Bromorophenyl)-4-oxo- $N, N^{\prime}$-di(pyridin-2-yl)cyclohexane-1,1-dicarboxamide (3e). Yield $90 \mathrm{mg}$ (0.14 mmol, 56\%); m.p. $227-228{ }^{\circ} \mathrm{C} ;{ }^{1} \mathrm{H}-\mathrm{NMR}$ (DMSO- $\left.d_{6}, 400 \mathrm{MHz}\right) \delta: 2.31$ and 2.34 (dd, $2 \mathrm{H}, J=4.4 \mathrm{~Hz}$ and $\left.12.0 \mathrm{~Hz}, \mathrm{CH}_{2}\right), 2.69(\mathrm{t}, 2 \mathrm{H}, J=12.4 \mathrm{~Hz}, \mathrm{CH}), 4.00$ and $4.02\left(\mathrm{dd}, 2 \mathrm{H}, J=4.4 \mathrm{~Hz}\right.$ and $\left.10.8 \mathrm{~Hz}, \mathrm{CH}_{2}\right)$, $6.93(\mathrm{t}, 1 \mathrm{H}, J=6.0 \mathrm{~Hz}, \mathrm{Ar}-\mathrm{H}), 7.00(\mathrm{~s}, 1 \mathrm{H}, \mathrm{NH}), 7.31(\mathrm{~d}, 4 \mathrm{H}, J=8.4 \mathrm{~Hz}, \mathrm{Ar}-\mathrm{H}) ; 7.39(\mathrm{~d}, 4 \mathrm{H}, J=8.4 \mathrm{~Hz}$, Ar-H), $7.49(\mathrm{t}, 1 \mathrm{H}, J=6.0 \mathrm{~Hz}, \mathrm{Ar}-\mathrm{H}), 7.52(\mathrm{~d}, 1 \mathrm{H}, J=8.0 \mathrm{~Hz}, \mathrm{ArH}), 7.61(\mathrm{t}, 1 \mathrm{H}, J=8.0 \mathrm{~Hz}, \mathrm{Ar}-\mathrm{H}), 7.78(\mathrm{~d}$, $1 \mathrm{H}, J=8.0 \mathrm{~Hz}, \mathrm{Ar}-\mathrm{H}), 7.91-8.03(\mathrm{~m}, 2 \mathrm{H}, \mathrm{Ar}-\mathrm{H}), 8.69$ and $8.70(\mathrm{dd}, 1 \mathrm{H}, J=1.2 \mathrm{~Hz}$ and $3.6 \mathrm{~Hz}, \mathrm{Ar}-\mathrm{H})$, $11.11(\mathrm{~s}, 1 \mathrm{H}, \mathrm{NH}) ;{ }^{13} \mathrm{C}-\mathrm{NMR}$ (DMSO- $\left.d_{6}, 100 \mathrm{MHz}\right) \delta: 42.6,45.4,58.4,87.2,113.5,120.2,120.7,124.0$, $125.5,130.9,131.7,138.5,138.7,141.4,148.4,149.8,150.2,150.4,167.9,170.2 ; \mathrm{IR}\left(\mathrm{KBr}, \mathrm{cm}^{-1}\right) v_{\max }=3414$, 3055, 1687, 1651, 1589, 1574, 1531, 1487, 1467, 1435, 1402, 1297, 1158, 1071, 1010, 993, 838, 819, 773, 554; [Anal. Calcd. for $\mathrm{C}_{30} \mathrm{H}_{24} \mathrm{Br}_{2} \mathrm{~N}_{4} \mathrm{O}_{3}$ : C, 55.58; H, 3.73; N, 8.64; Found: $\mathrm{C}, 55.71 ; \mathrm{H}, 3.86 ; \mathrm{N}, 8.53$ ]; LC/MS (ESI, $m / z$ ): $[\mathrm{M}+]$, found $646.00 \mathrm{C}_{30} \mathrm{H}_{24} \mathrm{Br}_{2} \mathrm{~N}_{4} \mathrm{O}_{3}$ for 646.02.

$N, N^{\prime}$-bis(5-Chloropyridin-2-yl)-2,6-bis(3-nitrophenyl)-4-oxocyclohexane-1,1-dicarboxamide (3f). Yield $100 \mathrm{mg}$ (0.15 mmol, 62\%); m.p. $174-175^{\circ} \mathrm{C} ;{ }^{1} \mathrm{H}-\mathrm{NMR}$ (DMSO- $\left.d_{6}, 400 \mathrm{MHz}\right) \delta: 2.29$ and $2.32(\mathrm{dd}, 2 \mathrm{H}$, $J=5.6 \mathrm{~Hz}$ and $\left.12.8 \mathrm{~Hz}, \mathrm{CH}_{2}\right), 2.74(\mathrm{t}, 1 \mathrm{H}, J=513.2 \mathrm{~Hz}, \mathrm{CH}), 3.02(\mathrm{t}, 1 \mathrm{H}, J=13.2 \mathrm{~Hz}, \mathrm{CH}), 4.06$ and 4.09 $\left(\mathrm{dd}, 1 \mathrm{H}, J=6.0 \mathrm{~Hz}\right.$ and $\left.10.1 \mathrm{~Hz}, \mathrm{CH}_{2}\right), 4.28$ and $4.31\left(\mathrm{dd}, 1 \mathrm{H}, J=6.4 \mathrm{~Hz}\right.$ and $\left.10.1 \mathrm{~Hz}, \mathrm{CH}_{2}\right), 7.17(\mathrm{~s}$, $1 \mathrm{H}, \mathrm{NH}), 7.33(\mathrm{~d}, 1 \mathrm{H}, J=8.8 \mathrm{~Hz}, \mathrm{Ar}-\mathrm{H}), 7.47(\mathrm{t}, 1 \mathrm{H}, J=7.6 \mathrm{~Hz}, \mathrm{Ar}-\mathrm{H}), 7.55(\mathrm{~d}, 1 \mathrm{H}, J=8.4 \mathrm{~Hz}, \mathrm{Ar}-\mathrm{H})$, 7.66-7.70 (m, 2H, Ar-H), $7.75(\mathrm{~d}, 1 \mathrm{H}, J=8.0 \mathrm{~Hz}, \mathrm{Ar}-\mathrm{H}), 7.94$ and $7.96(\mathrm{dd}, 1 \mathrm{H}, J=2.0 \mathrm{~Hz}$ and $8.0 \mathrm{~Hz}$, Ar-H), $8.02(\mathrm{~d}, 2 \mathrm{H}, J=7.6 \mathrm{~Hz}, \mathrm{Ar}-\mathrm{H}), 8.15-8.20(\mathrm{~m}, 3 \mathrm{H}, \mathrm{Ar}-\mathrm{H}), 8.27(\mathrm{~d}, 1 \mathrm{H}, J=2.4 \mathrm{~Hz}, \mathrm{Ar}-\mathrm{H}), 8.70(\mathrm{~d}, 1 \mathrm{H}$, $J=2.4 \mathrm{~Hz}, \mathrm{Ar}-\mathrm{H}), 10.78(\mathrm{~s}, 1 \mathrm{H}, \mathrm{NH}) ;{ }^{13} \mathrm{C}-\mathrm{NMR}$ (DMSO- $\left.d_{6}, 100 \mathrm{MHz}\right) \delta: 43.9,44.6,59.1,87.4,114.9,122.0$, 123.0, 124.4, 124.5, 126.0, 126.7, 130.0, 130.4, 131.0, 133.7, 135.9, 138.2, 138.4, 141.5, 145.8, 146.9, 147.8, 148.0, 148.1, 148.8, 149.6, 158.4, 168.5, 170.7; IR $\left(\mathrm{KBr}_{\mathrm{cm}}{ }^{-1}\right) v_{\max }=3236,3087,1733,1693,1650,1568$, $1528,1463,1374,1351,1286,1240,1170,1114,1015,826,806,686$; [Anal. Calcd. for $\mathrm{C}_{30} \mathrm{H}_{22} \mathrm{Cl}_{2} \mathrm{~N}_{6} \mathrm{O}_{7}$ : C, 55.48; H, 3.41; N, 12.94; Found: C, 55.62; H, 3.54; N, 13.08; LC/MS (ESI, $m / z)$ : [M+], found 648.10; $\mathrm{C}_{30} \mathrm{H}_{22} \mathrm{Cl}_{2} \mathrm{~N}_{6} \mathrm{O}_{7}$ for 648.09 .

$N, N^{\prime}$-bis(5-Chloropyridin-2-yl)-2,6-bis(4-methoxyphenyl)-4-oxocyclohexane-1,1-dicarboxamide (3g). Yield $110 \mathrm{mg}(0.18 \mathrm{mmol}, 72 \%)$; m.p. $203-204{ }^{\circ} \mathrm{C} ;{ }^{1} \mathrm{H}-\mathrm{NMR}$ (DMSO- $d_{6}, 400 \mathrm{MHz}$ ) $\delta: 2.30$ and 2.33 (dd, 
$2 \mathrm{H}, J=5.2 \mathrm{~Hz}$ and $\left.11.6 \mathrm{~Hz}, \mathrm{CH}_{2}\right), 2.66(\mathrm{t}, 2 \mathrm{H}, J=11.6 \mathrm{~Hz}, \mathrm{CH}), 3.58\left(\mathrm{~s}, 6 \mathrm{H}, \mathrm{OCH}_{3}\right), 3.91$ and $3.94(\mathrm{dd}$, $2 \mathrm{H}, J=4.8 \mathrm{~Hz}$ and $\left.11.6 \mathrm{~Hz}, \mathrm{CH}_{2}\right), 6.72(\mathrm{~d}, 4 \mathrm{H}, J=9.6 \mathrm{~Hz}, \mathrm{Ar}-\mathrm{H}), 7.02(\mathrm{~s}, 1 \mathrm{H}, \mathrm{NH}), 7.22(\mathrm{~d}, 4 \mathrm{H}, J=9.6$ $\mathrm{Hz}, \mathrm{Ar}-\mathrm{H}), 7.57(\mathrm{~d}, 1 \mathrm{H}, J=9.6 \mathrm{~Hz}, \mathrm{Ar}-\mathrm{H}), 7.71$ and $7.73(\mathrm{dd}, 1 \mathrm{H}, J=2.4 \mathrm{~Hz}$ and $8.8 \mathrm{~Hz}, \mathrm{Ar}-\mathrm{H}), 7.84$ $(\mathrm{d}, 1 \mathrm{H}, J=9.2 \mathrm{~Hz}, \mathrm{Ar}-\mathrm{H}), 8.02(\mathrm{~d}, 1 \mathrm{H}, J=2.8 \mathrm{~Hz}, \mathrm{Ar}-\mathrm{H}), 8.13$ and $8.15(\mathrm{dd}, 1 \mathrm{H}, J=2.8 \mathrm{~Hz}$ and $8.0 \mathrm{~Hz}$, Ar-H), $8.77(\mathrm{~d}, 1 \mathrm{H}, J=2.4 \mathrm{~Hz}, \mathrm{Ar}-\mathrm{H}), 11.16(\mathrm{~s}, 1 \mathrm{H}, \mathrm{NH}){ }^{13} \mathrm{C}-\mathrm{NMR}$ (DMSO- $\left.d_{6}, 100 \mathrm{MHz}\right) \delta: 40.5,45.2$, 55.3, 59.3, 87.6, 114.1, 114.6, 125.6, 126.7, 129.7, 130.9, 133.9, 138.2, 138.3, 146.6, 148.3, 148.8, 149.2, 158.4, 168.5, 170.7; IR $\left(\mathrm{KBr}, \mathrm{cm}^{-1}\right) v_{\max }=3124,2960,2833,1693,1656,1610,1569,1512,1460,1409,1376,1305$, 1251, 1177, 1156, 1107, 1031, 1015, 837, 574; [Anal. Calcd. for $\mathrm{C}_{32} \mathrm{H}_{28} \mathrm{Cl}_{2} \mathrm{~N}_{4} \mathrm{O}_{5}$ : C, 62.04; $\mathrm{H}, 4.56$; N, 9.04; Found: $\mathrm{C}, 61.87 ; \mathrm{H}, 4.45 ; \mathrm{N}, 9.19$; LC/MS (ESI, $\mathrm{m} / \mathrm{z}$ ): [M+], found $618.14 \mathrm{C}_{32} \mathrm{H}_{28} \mathrm{Cl}_{2} \mathrm{~N}_{4} \mathrm{O}_{5}$ for 618.14 .

N,N'-bis(5-Chloropyridin-2-yl)-2,6-di(naphthalen-2-yl)-4-oxocyclohexane-1,1-dicarboxamide (3h). Yield $95 \mathrm{mg}$ (0.14 mmol, 58\%); m.p. $114-115{ }^{\circ} \mathrm{C} ;{ }^{1} \mathrm{H}-\mathrm{NMR}$ (DMSO- $\left.d_{6}, 400 \mathrm{MHz}\right) \delta: 2.22-2.27$ (m, $\left.2 \mathrm{H}, \mathrm{CH}_{2}\right), 3.07(\mathrm{t}, 2 \mathrm{H}, J=12.0 \mathrm{~Hz}, \mathrm{CH}), 5.24-5.27\left(\mathrm{~m}, 2 \mathrm{H}, \mathrm{CH}_{2}\right), 6.80(\mathrm{~d}, 1 \mathrm{H}, J=9.2 \mathrm{~Hz}, \mathrm{Ar}-\mathrm{H}), 7.07$ $(\mathrm{s}, 1 \mathrm{H}, \mathrm{NH}), 7.26$ and $7.28(\mathrm{dd}, 1 \mathrm{H}, J=2.4 \mathrm{~Hz}$ and $8.8 \mathrm{~Hz}, \mathrm{Ar}-\mathrm{H}), 7.39-7.47(\mathrm{~m}, 5 \mathrm{H}, \mathrm{Ar}-\mathrm{H}), 7.58(\mathrm{t}, 2 \mathrm{H}$, $J=8.0 \mathrm{~Hz}, \mathrm{Ar}-\mathrm{H}), 7.66(\mathrm{~d}, 2 \mathrm{H}, J=8.4 \mathrm{~Hz}, \mathrm{Ar}-\mathrm{H}), 7.72(\mathrm{~d}, 2 \mathrm{H}, J=8.4 \mathrm{~Hz}, \mathrm{Ar}-\mathrm{H}), 7.76(\mathrm{~d}, 2 \mathrm{H}, J=8.4 \mathrm{~Hz}$, Ar-H), $7.92(\mathrm{~d}, 1 \mathrm{H}, J=2.8 \mathrm{~Hz}, \mathrm{Ar}-\mathrm{H}), 8.10$ and $8.12(\mathrm{dd}, 1 \mathrm{H}, J=2.8 \mathrm{~Hz}$ and $8.8 \mathrm{~Hz}, \mathrm{Ar}-\mathrm{H}), 8.47(\mathrm{~d}, 2 \mathrm{H}$, $J=8.4 \mathrm{~Hz}, \mathrm{Ar}-\mathrm{H}), 8.77(\mathrm{~d}, 1 \mathrm{H}, J=2.8 \mathrm{~Hz}, \mathrm{Ar}-\mathrm{H}), 11.13(\mathrm{~s}, 1 \mathrm{H}, \mathrm{NH}) ;{ }^{13} \mathrm{C}-\mathrm{NMR}$ (DMSO- $\left.d_{6}, 100 \mathrm{MHz}\right) \delta$ : 40.7(merged with dmso- $d_{6}$ ), 44.4, 57.0, 87.5, 114.0, 123.5, 124.5, 125.3, 126.0, 126.1, 126.3, 126.6, 127.6, $128.8,131.1,131.7,133.7,137.5,138.5,140.2,146.4,148.4,148.8,148.9,168.1,172.3 ; \mathrm{IR}\left(\mathrm{KBr}_{\mathrm{cm}}{ }^{-1}\right) v_{\max }$ $=3234,3085,1737,1692,1654,1569,1521,1464,1377,1359,1287,1248,1173,1113,1018,824,807$; [Anal. Calcd. for $\mathrm{C}_{38} \mathrm{H}_{28} \mathrm{Cl}_{2} \mathrm{~N}_{4} \mathrm{O}_{3}$ : C, 69.20; H, 4.28; N, 8.49; Found: C, 69.11; H, 4.19; N, 8.67; LC/MS (ESI, $\mathrm{m} / \mathrm{z}$ ): $[\mathrm{M}+]$, found $658.10 ; \mathrm{C}_{38} \mathrm{H}_{28} \mathrm{Cl}_{2} \mathrm{~N}_{4} \mathrm{O}_{3}$ for 658.15.

$N, N^{\prime}$-bis(5-Chloropyridin-2-yl)-4-oxo-2,6-di(thiophen-2-yl)cyclohexane-1,1-dicarboxamide (3i). Yield $85 \mathrm{mg}(0.15 \mathrm{mmol}, 60 \%)$; m.p. $165-166{ }^{\circ} \mathrm{C} ;{ }^{1} \mathrm{H}-\mathrm{NMR}$ (DMSO- $\left.d_{6}, 400 \mathrm{MHz}\right) \delta: 2.36(\mathrm{~d}, 2 \mathrm{H}, J=11.6 \mathrm{~Hz}$, $\left.\mathrm{CH}_{2}\right), 2.75(\mathrm{t}, 2 \mathrm{H}, J=11.6 \mathrm{~Hz}, \mathrm{CH}), 4.26$ and $4.28\left(\mathrm{~m}, 2 \mathrm{H}, J=4.4 \mathrm{~Hz}\right.$ and $\left.10.0 \mathrm{~Hz}, \mathrm{CH}_{2}\right), 6.83(\mathrm{t}, 2 \mathrm{H}$, $J=4.0 \mathrm{~Hz}, \mathrm{Ar}-\mathrm{H}), 6.99(\mathrm{~d}, 2 \mathrm{H}, J=2.4 \mathrm{~Hz}, \mathrm{Ar}-\mathrm{H}), 7.13(\mathrm{~s}, 1 \mathrm{H}, \mathrm{NH}), 7.24(\mathrm{~d}, 2 \mathrm{H}, J=4.4 \mathrm{~Hz}, \mathrm{Ar}-\mathrm{H}), 7.48(\mathrm{~d}$, $1 \mathrm{H}, J=8.4 \mathrm{~Hz}, \mathrm{Ar}-\mathrm{H}), 7.80(\mathrm{~d}, 1 \mathrm{H}, J=11.2 \mathrm{~Hz}, \mathrm{Ar}-\mathrm{H}), 7.99(\mathrm{~d}, 1 \mathrm{H}, J=8.4 \mathrm{~Hz}, \mathrm{Ar}-\mathrm{H}), 8.09(\mathrm{~s}, 1 \mathrm{H}, \mathrm{Ar}-\mathrm{H})$, $8.13(\mathrm{~d}, 1 \mathrm{H}, J=7.2 \mathrm{~Hz}, \mathrm{Ar}-\mathrm{H}), 8.73(\mathrm{~s}, 1 \mathrm{H}, \mathrm{Ar}-\mathrm{H}), 11.31(\mathrm{~s}, 1 \mathrm{H}, \mathrm{NH}) ;{ }^{13} \mathrm{C}-\mathrm{NMR}$ (DMSO- $\left.d_{6}, 100 \mathrm{MHz}\right) \delta$ : 40.5 (merged with dmso- $d_{6}$ ), 40.5, 43.9, 59.9, 87.4, 114.8, 125.5, 125.9, 126.1, 126.5, 127.4, 131.0, 138.3, 138.4, 144.7, 146.8, 148.3, 148.6, 149.3, 168.5, 170.0; IR $\left(\mathrm{KBr}, \mathrm{cm}^{-1}\right) v_{\max }=3426,3236,2951,2925,1687$, 1569, 1530, 1461, 1374, 1291, 1155, 1111, 1015, 851, 835, 696, 582; [Anal. Calcd. for $\mathrm{C}_{26} \mathrm{H}_{20} \mathrm{Cl}_{2} \mathrm{~N}_{4} \mathrm{O}_{3} \mathrm{~S}_{2}$ : C, 54.64; H, 3.53; N, 9.80; Found: C, 54.72; H, 3.41; N, 9.97; LC/MS (ESI, $m / z)$ : [M+], found 570.00; $\mathrm{C}_{26} \mathrm{H}_{20} \mathrm{Cl}_{2} \mathrm{~N}_{4} \mathrm{O}_{3} \mathrm{~S}_{2}$ for 570.04 .

$N, N^{\prime}$-bis(5-Chloropyridin-2-yl)-2,6-di(furan-2-yl)-4-oxocyclohexane-1,1-dicarboxamide (3j). Yield $70 \mathrm{mg}(0.13 \mathrm{mmol}, 52.0 \%)$; m.p. $160-161{ }^{\circ} \mathrm{C} ;{ }^{1} \mathrm{H}-\mathrm{NMR}$ (DMSO- $\left.d_{6}, 400 \mathrm{MHz}\right) \delta: 2.27$ and 2.29 (dd, $2 \mathrm{H}$, $J=4.4 \mathrm{~Hz}$ and $\left.11.2 \mathrm{~Hz}, \mathrm{CH}_{2}\right), 2.57(\mathrm{t}, 2 \mathrm{H}, J=11.2 \mathrm{~Hz}, \mathrm{CH}), 4.07$ and $4.10(\mathrm{dd}, 2 \mathrm{H}, J=4.8 \mathrm{~Hz}$ and $10.8 \mathrm{~Hz}$, $\left.\mathrm{CH}_{2}\right), 6.20(\mathrm{~d}, 2 \mathrm{H}, J=2.8 \mathrm{~Hz}, \mathrm{Ar}-\mathrm{H}), 6.23-6.24(\mathrm{~m}, 2 \mathrm{H}, \mathrm{Ar}-\mathrm{H}), 7.07(\mathrm{~s}, 1 \mathrm{H}, \mathrm{NH}), 7.37(\mathrm{~d}, 1 \mathrm{H}, J=8.4 \mathrm{~Hz}$, Ar-H), $7.40(\mathrm{~s}, 2 \mathrm{H}, \mathrm{Ar}-\mathrm{H}), 7.81$ and $7.83(\mathrm{dd}, 1 \mathrm{H}, J=2.8 \mathrm{~Hz}$ and $8.8 \mathrm{~Hz}, \mathrm{Ar}-\mathrm{H}), 8.01(\mathrm{~d}, 1 \mathrm{H}, J=8.4 \mathrm{~Hz}$, Ar-H), 8.05 and $8.08(\mathrm{dd}, 1 \mathrm{H}, J=2.4 \mathrm{~Hz}$ and $8.4 \mathrm{~Hz}, \mathrm{Ar}-\mathrm{H}), 8.15(\mathrm{~d}, 1 \mathrm{H}, J=2.8 \mathrm{~Hz}, \mathrm{Ar}-\mathrm{H}), 8.67(\mathrm{~d}, 1 \mathrm{H}$, $J=2.8 \mathrm{~Hz}, \mathrm{Ar}-\mathrm{H}), 11.40(\mathrm{~s}, 1 \mathrm{H}, \mathrm{NH}) ;{ }^{13} \mathrm{C}-\mathrm{NMR}$ (DMSO- $\left.d_{6}, 100 \mathrm{MHz}\right) \delta: 38.7,39.3,40.6,55.9,87.1,107.3$, $110.9,114.8,125.8,126.4,130.8,138.2,138.4,143.0,146.8,148.0,148.6,149.6,154.6,168.3,169.9$; IR (KBr, $\mathrm{cm}^{-1}$ ) $v_{\max }=3420,3243,2952,1691,1630,1569,1530,1463,1418,1375,1291,1163,1112,1014,809$, 735; [Anal. Calcd. for $\mathrm{C}_{26} \mathrm{H}_{20} \mathrm{Cl}_{2} \mathrm{~N}_{4} \mathrm{O}_{5}: \mathrm{C}, 57.90 ; \mathrm{H}, 3.74 ; \mathrm{N}, 13.15$; Found: $\mathrm{C}, 58.11 ; \mathrm{H}, 3.63 ; \mathrm{N}, 12.89$; LC/MS (ESI, $m / z$ ): [M+], found 538.1; $\mathrm{C}_{26} \mathrm{H}_{20} \mathrm{Cl}_{2} \mathrm{~N}_{4} \mathrm{O}_{5}$ for 538.08 .

2,6-bis(3-Bromophenyl)- $N, N^{\prime}$-bis(5-chloropyridin-2-yl)-4-oxocyclohexane-1,1-dicarboxamide (3k). Yield $78 \mathrm{mg}(0.11 \mathrm{mmol}, 44.0 \%)$; m.p. $213-214{ }^{\circ} \mathrm{C} ;{ }^{1} \mathrm{H}-\mathrm{NMR}$ (DMSO- $\left.d_{6}, 400 \mathrm{MHz}\right) \delta: 2.32$ and 2.35 (dd, $2 \mathrm{H}, J=4.8 \mathrm{~Hz}$ and $\left.11.6 \mathrm{~Hz}, \mathrm{CH}_{2}\right), 2.69(\mathrm{t}, 2 \mathrm{H}, J=11.2 \mathrm{~Hz}, \mathrm{CH}), 4.00$ and $4.04(\mathrm{dd}, 2 \mathrm{H}, J=5.2 \mathrm{~Hz}$ and $\left.10.8 \mathrm{~Hz}, \mathrm{CH}_{2}\right), 7.15(\mathrm{~s}, 1 \mathrm{H}, \mathrm{NH}), 7.17(\mathrm{t}, 2 \mathrm{H}, J=8.0 \mathrm{~Hz}, \mathrm{Ar}-\mathrm{H}), 7.30(\mathrm{t}, 4 \mathrm{H}, J=8.0 \mathrm{~Hz}, \mathrm{Ar}-\mathrm{H}), 7.53(\mathrm{~s}, 2 \mathrm{H}$, Ar-H), $7.56(\mathrm{~d}, 1 \mathrm{H}, J=8.0 \mathrm{~Hz}, \mathrm{Ar}-\mathrm{H}), 7.76$ and $7.78(\mathrm{dd}, 1 \mathrm{H}, J=2.0 \mathrm{~Hz}$ and $8.8 \mathrm{~Hz}, \mathrm{Ar}-\mathrm{H}), 7.83(\mathrm{~d}, 1 \mathrm{H}$, 
$J=8.0 \mathrm{~Hz}, \mathrm{Ar}-\mathrm{H}), 8.05(\mathrm{~d}, 1 \mathrm{H}, J=2.8 \mathrm{~Hz}, \mathrm{Ar}-\mathrm{H}), 8.18$ and $8.20(\mathrm{dd}, 1 \mathrm{H}, J=2.8 \mathrm{~Hz}$ and $8.0 \mathrm{~Hz}, \mathrm{Ar}-\mathrm{H})$, $8.74(\mathrm{~d}, 1 \mathrm{H}, J=2.4 \mathrm{~Hz}, \mathrm{Ar}-\mathrm{H}), 11.09(\mathrm{~s}, 1 \mathrm{H}, \mathrm{NH}),{ }^{13} \mathrm{C}-\mathrm{NMR}$ (DMSO- $\left.d_{6}, 100 \mathrm{MHz}\right) \delta: 42.2,45.4,58.8,87.6$, 114.5, 121.9, 126.0, 126.6, 127.4, 130.5, 131.0, 131.2, 131.8, 138.3, 138.6, 144.3, 146.8, 148.2, 148.6, 148.8, 167.9, 170.3; IR $\left(\mathrm{KBr}, \mathrm{cm}^{-1}\right) v_{\max }=3386,3249,2930,1688,1657,1569,1519,1460,1372,1305,1286,1153$, 1114, 1009, 834, 804, 696; [Anal. Calcd. for $\mathrm{C}_{30} \mathrm{H}_{22} \mathrm{Br}_{2} \mathrm{Cl}_{2} \mathrm{~N}_{4} \mathrm{O}_{3}$ : C, 50.24; H, 3.09; N, 7.81; Found: C, 50.07; H, 3.26; N, 7.92; LC/MS (ESI, $m / z)$ : [M+], found 714.00; $\mathrm{C}_{30} \mathrm{H}_{22} \mathrm{Br}_{2} \mathrm{Cl}_{2} \mathrm{~N}_{4} \mathrm{O}_{3}$ for 713.94.

N,N'-bis(5-Chloropyridin-2-yl)-4-oxo-2,6-bis(4-(trifluoromethyl)phenyl)cyclohex-ane-1,1-dicarboxamide (31). Yield $64 \mathrm{mg}(0.92 \mathrm{mmol}, 37.0 \%)$; m.p. $138-139{ }^{\circ} \mathrm{C} ;{ }^{1} \mathrm{H}-\mathrm{NMR}$ (DMSO- $d_{6}, 400 \mathrm{MHz}$ ) $\delta: 2.36$ and 2.39 $\left(\mathrm{dd}, 2 \mathrm{H}, J=4.4 \mathrm{~Hz}\right.$ and $\left.11.6 \mathrm{~Hz}, \mathrm{CH}_{2}\right), 2.75(\mathrm{t}, 2 \mathrm{H}, J=11.2 \mathrm{~Hz}, \mathrm{CH}), 4.14$ and $4.16(\mathrm{dd}, 2 \mathrm{H}, J=5.2 \mathrm{~Hz}$ and $\left.10.8 \mathrm{~Hz}, \mathrm{CH}_{2}\right), 7.23(\mathrm{~s}, 1 \mathrm{H}, \mathrm{NH}), 7.53-7.57(\mathrm{~m}, 9 \mathrm{H}, \mathrm{Ar}-\mathrm{H}), 7.67(\mathrm{~d}, 1 \mathrm{H}, J=8.8 \mathrm{~Hz}, \mathrm{Ar}-\mathrm{H}), 7.72-7.43$ $(\mathrm{m}, 1 \mathrm{H}, \mathrm{Ar}-\mathrm{H}), 7.98-7.99(\mathrm{~m}, 1 \mathrm{H}, \mathrm{Ar}-\mathrm{H}), 8.15$ and $8.18(\mathrm{dd}, 1 \mathrm{H}, J=2.8 \mathrm{~Hz}$ and $8.4 \mathrm{~Hz}, \mathrm{Ar}-\mathrm{H}), 8.77(\mathrm{~d}, 1 \mathrm{H}$, $J=2.8 \mathrm{~Hz}, \mathrm{Ar}-\mathrm{H}), 10.99$ (s, $1 \mathrm{H}, \mathrm{NH}) ;{ }^{13} \mathrm{C}-\mathrm{NMR}$ (DMSO-d $\left.d_{6}, 100 \mathrm{MHz}\right) \delta: 42.0,45.8,58.6,87.6,114.3$, 123.1, 125.7, 125.9, 126.9, 128.0, 128.4, 129.5, 131.2, 138.2, 138.5, 146.4, 146.7, 148.4, 148.5, 148.7, 167.7, 170.2; IR $\left(\mathrm{KBr}, \mathrm{cm}^{-1}\right) v_{\max }=3195,2956,1693,1653,1570,1523,1463,1399,1376,1324,1163,1124,1068$, 1017, 841, 609; [Anal. Calcd. for $\mathrm{C}_{32} \mathrm{H}_{22} \mathrm{Cl}_{2} \mathrm{~F}_{6} \mathrm{~N}_{4} \mathrm{O}_{3}$ : C, 55.27; H, 3.19; N, 8.06; Found: C, 55.13; H, 3.39; $\mathrm{N}$, 8.17; LC/MS (ESI, $m / z)$ : [M+], found 694.10; $\mathrm{C}_{32} \mathrm{H}_{22} \mathrm{Cl}_{2} \mathrm{~F}_{6} \mathrm{~N}_{4} \mathrm{O}_{3}$ for 694.10.

2,6-bis(4-Fluorophenyl)-4-oxo- $N, N^{\prime}$-di(pyridin-2-yl)cyclohexane-1,1-dicarboxamide (3m). Yield $112 \mathrm{mg}$ (0.21 mmol, 85\%); m.p. $245-246{ }^{\circ} \mathrm{C} ;{ }^{1} \mathrm{H}-\mathrm{NMR}$ (DMSO- $\left.d_{6}, 400 \mathrm{MHz}\right) \delta: 2.32$ and $2.35(\mathrm{dd}, 2 \mathrm{H}, J=4.4 \mathrm{~Hz}$ and $\left.11.2 \mathrm{~Hz}, \mathrm{CH}_{2}\right), 2.69(\mathrm{t}, 2 \mathrm{H}, J=12.0 \mathrm{~Hz}, \mathrm{CH}), 4.07$ and $4.04\left(\mathrm{~m}, 2 \mathrm{H}, \mathrm{CH}_{2}\right), 6.89-6.92(\mathrm{~m}, 1 \mathrm{H}, \mathrm{Ar}-\mathrm{H})$, $6.93(\mathrm{~s}, 1 \mathrm{H}, \mathrm{NH}), 7.02(\mathrm{t}, 4 \mathrm{H}, J=9.2 \mathrm{~Hz}, \mathrm{Ar}-\mathrm{H}), 7.37-7.41(\mathrm{~m}, 4 \mathrm{H}, \mathrm{Ar}-\mathrm{H}), 7.48-7.51(\mathrm{~m}, 1 \mathrm{H}, \mathrm{Ar}-\mathrm{H})$, 7.53-7.56 (m, 1H, ArH), 7.57-7.61 (m, 1H, Ar-H), 7.76-7.78 (m, 1H, Ar-H), 7.96-7.98 (m, 1H, Ar-H), 8.01 $(\mathrm{dt}, 1 \mathrm{H}, J=2.0 \mathrm{~Hz}$ and $8.0 \mathrm{~Hz}, \mathrm{Ar}-\mathrm{H}), 8.69-8.71(\mathrm{~m}, 1 \mathrm{H}, \mathrm{Ar}-\mathrm{H}), 11.11(\mathrm{~s}, 1 \mathrm{H}, \mathrm{NH}) ;{ }^{13} \mathrm{C}-\mathrm{NMR}$ (DMSO- $d_{6}$, $100 \mathrm{MHz}$ ) $\delta: 42.9,45.2,58.8,87.2,113.5,115.4,115.6,120.1,123.9,125.5,130.6,130.7,138.2,138.3,138.4$, $138.6,148.3,149.8,150.3,150.5,160.3,162.7,168.1,170.3 ; \mathrm{IR}\left(\mathrm{KBr}_{\mathrm{cm}}{ }^{-1}\right) v_{\max }=3421,3065,1678,1655$, 1589, 1576, 1533, 1488, 1462, 1434, 1408, 1291, 1154, 1077, 1011, 993, 839, 815, 770, 559; [Anal. Calcd. for $\mathrm{C}_{30} \mathrm{H}_{24} \mathrm{~F}_{2} \mathrm{~N}_{4} \mathrm{O}_{3}$ : C, 68.43; H, 4.59; N, 10.64; Found: C, 68.57; H, 4.71; N, 10.42]; LC/MS (ESI, $\left.m / z\right):$ [M+], found $526.20 \mathrm{C}_{30} \mathrm{H}_{24} \mathrm{~F}_{2} \mathrm{~N}_{4} \mathrm{O}_{3}$ for 526.18 .

\subsection{Anticancer Activity}

\subsubsection{Cell Lines and Drugs}

The cytotoxic activity of the new synthesized compounds was tested in different mammalian cancer cells, breast cancer (+ve ER) (MCF-7), breast cancer (-ve ER) (MDA-MB-231), tongue (oral cancer) (SAS), prostate cancer (PC-3), colorectal cancer (HCT-116) and hepatocellular carcinoma (HuH-7 and HepG-2). The cell lines were obtained from the American Type Culture Collection (ATCC). The cells were cultivated at $37{ }^{\circ} \mathrm{C}$ and $5 \% \mathrm{CO}_{2}$ in DMEM (Lonza) medium supplemented with $10 \%$ fetal bovine serum (Lonza), $100 \mathrm{IU} / \mathrm{mLpenicillin} \mathrm{and} 100 \mu \mathrm{g} / \mathrm{mL}$ streptomycin (Lonza). Cisplatin was used as a positive control and was obtained from Sigma-Aldrich. The synthesized compounds were solubilized in DMSO and stored at $-20^{\circ} \mathrm{C}$. For the initial screening, $0.5 \%$ crystal violet was used [21]. The viability of the cells were determined by using the MTT reagent $[22,23]$.

\subsubsection{Cytotoxicity Assay}

"The cells were seeded in a 96-well plate and serial dilutions of the tested compounds or cisplatin was added after overnight incubation of the cells at $37^{\circ} \mathrm{C}$ and $5 \% \mathrm{CO}_{2}$. DMSO was used as a negative control $(0.1 \%)$. After that, MTT ( $5 \mathrm{mg} / \mathrm{mL}$ PBS) was added after 48 hours of incubation. The formazan crystals were solubilized by the acidified SDS solution. The absorbance was recorded at $570 \mathrm{~nm}$ by Biotech ELx- $800^{\mathrm{TM}}$ plate reader (Winooski, VT, USA). The viability assay was performed 3 times and the standard deviation was determined $( \pm)$. $\mathrm{IC}_{50}$ was calculated as the concentration that causes $50 \%$ inhibition of cell growth. The selectivity index was calculated as previously reported" [26,27]. 


\subsection{3. $\alpha$-Glucosidase Inhibitory Assay}

"Certain aliquots $(40 \mu \mathrm{L}$ ) of compounds (prepared in 50\% DMSO and 50\% water) at different concentrations (3-500 $\mu \mathrm{g} / \mathrm{mL})$ were pre-incubated with a potassium phosphate buffer $(80 \mu \mathrm{L}, \mathrm{pH}$ 6.8), containing $67 \mathrm{mM}$ potassium phosphate and $2.0 \mathrm{unit} / \mathrm{ml} \alpha$-glucosidase in a 96-well plate for $10 \mathrm{~min}$. After that, $40 \mu \mathrm{L}$ of $5 \mathrm{mM}$ p-nitrophenyl- $\alpha$-D-glucopyranoside solution (p-NPG) in potassium phosphate buffer was added into the mixture and incubated for another $10 \mathrm{~min}$. After incubation, $100 \mathrm{mM} \mathrm{Na}_{2} \mathrm{CO}_{3}(60 \mu \mathrm{L})$ was added into the mixture to terminate the reaction and the absorbance of the mixture was measured at a wavelength of $415 \mathrm{~nm}$. The experiment was also carried out using a standard inhibitor, namely acarbose (positive control). The concentration resulting in $50 \%$ inhibition of $\alpha$-glucosidase activity $\left(\mathrm{IC}_{50}\right)$ was determined by using GraphPad Prism 5 statistical package (GraphPad ${ }^{\circledR}$ Software Inc., San Diego, CA, USA). All data were expressed as means \pm standard deviations of triplicate determinations" [28].

\subsubsection{Molecular Docking Study}

The docking studies were performed using the OpenEye Modeling software (License 2018-2019, OpenEye Scientific, NM, USA) [29-31]. A virtual library of the target compounds was used and their energies were minimized using the MMFF94 force field, followed by the generation of multi-conformers using the OMEGA application. The whole library of minimized energy values was used to dock an appropriate target according to the reported crystalized standard. The receptor PDB files for EGFR were downloaded from the Protein Data Bank (PDB:ID: 1M17). Both the ligand input file and the receptor input file were used as the input into FRED to perform the molecular docking simulations. Multiple scoring functions were employed to predict the energy profile of the ligand-receptor complex. The VIDA application was employed as a visualization tool to show the pose of the ligands and the potential binding interactions of the ligands to the receptor of interest.

\section{Conclusions}

The present study mainly focuses on the synthesis of a new series of pyridine-dicarboximidecyclohexanone-based chemical entities with improved anticancer activities. This new series was obtained via the DBU basic system, which exerts significant effects by promoting the Michael addition reaction. The synthesized compounds were screened against different cancer cell lines and were evaluated for their $\alpha$-glucosidase inhibitory activities. Consequently, the compounds $3 c, 3 e$ and 31 showed the most promising anticancer activities against different cancer cell lines. Thus, further studies are warranted to evaluate the underlying mechanism.

Author Contributions: Conceptualization, F.A.B. and A.B.; Data curation, A.F.M.M.R.; Formal analysis, F.F.E.-S. and F.A.B.; Funding acquisition, A.M.A.-M.; Investigation, M.S.I. and S.A.; Methodology, S.A.; Project administration, M.A.; Resources, M.A.; Software, Y.A.M.M.E.; Supervision, A.M.A.-M.; Validation, Y.A.M.M.E.; Visualization, A.B.; Writing-original draft, F.F.E.-S. and A.B.; Writing—review and editing, F.A.B. and A.B.

Funding: Deanship of Scientific Research at King Saud University, Riyadh, Saudi Arabia.

Acknowledgments: The authors would like to extend their sincere appreciation to the Deanship of Scientific Research at King Saud University for the funding granted to this research group (RGP-044). The authors would also like to thank the Deanship of Scientific Research and RSSU at King Saud University for their technical support.

Conflicts of Interest: The authors declare no conflict of interest.

\section{References}

1. Traoré, T.; Cavagnino, A.; Saettel, N.; Radvanyi, F.; Piguel, S.; Bernard-Pierrot, I.; Stoven, V.; Legraverend, M. New aminopyrimidine derivatives as inhibitors of the TAM family. Eur. J. Med. Chem. 2013, 70, $789-801$. [CrossRef] [PubMed]

2. Cohen, P.; Alessi, D.R. Kinase drug discovery-what's next in the field? ACS Chem. Biol. 2012, 8, 96-104. [CrossRef] [PubMed] 
3. Nakagawa, T.; Tohyama, O.; Yamaguchi, A.; Matsushima, T.; Takahashi, K.; Funasaka, S.; Shirotori, S.; Asada, M.; Obaishi, H. E7050: A dual c-Met and VEGFR-2 tyrosine kinase inhibitor promotes tumor regression and prolongs survival in mouse xenograft models. Cancer Sci. 2010, 101, 210-215. [CrossRef] [PubMed]

4. Squibb, B.-M. Multiple Ascending Dose Study of BMS-777607 in Subjects With Advanced or Metastatic Solid Tumors. Available online: https:/ / clinicaltrials.gov/ct2/show /NCT00605618 (accessed on 15 October 2018).

5. Dai, Y.; Siemann, D.W. BMS-777607, a Small-Molecule Met Kinase Inhibitor, Suppresses Hepatocyte Growth Factor-Stimulated Prostate Cancer Metastatic Phenotype In vitro. Mol. Cancer Ther. 2010, 1535-7163. [CrossRef] [PubMed]

6. Schroeder, G.M.; An, Y.; Cai, Z.-W.; Chen, X.-T.; Clark, C.; Cornelius, L.A.; Dai, J.; Gullo-Brown, J.; Gupta, A.; Henley, B. Discovery of N-(4-(2-amino-3-chloropyridin-4-yloxy)-3-fluorophenyl)4-ethoxy-1-(4-fluorophenyl)-2-oxo-1, 2-dihydropyridine-3-carboxamide (BMS-777607), a selective and orally efficacious inhibitor of the Met kinase superfamily. J. Med. Chem. 2009, 52, 1251-1254. [CrossRef]

7. Sharma, S.; Zeng, J.-Y.; Zhuang, C.-M.; Zhou, Y.-Q.; Yao, H.-P.; Hu, X.; Zhang, R.; Wang, M.-H. Small-molecule inhibitor BMS-777607 induces breast cancer cell polyploidy with increased resistance to cytotoxic chemotherapy agents. Mol. Cancer Ther. 2013, 12, 725-736. [CrossRef]

8. Chu, G.-H.; Gu, M.; Cassel, J.A.; Belanger, S.; Graczyk, T.M.; DeHaven, R.N.; Conway-James, N.; Koblish, M.; Little, P.J.; DeHaven-Hudkins, D.L. Novel malonamide derivatives as potent $\mathrm{k}$ opioid receptor agonists. Bioorg. Med. Chem. Lett. 2007, 17, 1951-1955. [CrossRef] [PubMed]

9. Islam, M.S.; Barakat, A.; Al-Majid, A.M.; Ghabbour, H.A.; Rahman, A.M.; Javaid, K.; Imad, R.; Yousuf, S.; Choudhary, M.I. A concise synthesis and evaluation of new malonamide derivatives as potential $\alpha$-glucosidase inhibitors. Biorg. Med. Chem. 2016, 24, 1675-1682. [CrossRef]

10. Barakat, A.; Islam, M.S.; Al-Majid, A.M.; Soliman, S.M.; Ghabbour, H.A.; Yousuf, S.; Choudhary, M.I.; Ul-Haq, Z. Synthesis, molecular structure, spectral analysis and biological activity of new malonamide derivatives as $\alpha$-glucosidase inhibitors. J. Mol. Struct. 2017, 1134, 253-264. [CrossRef]

11. Vranken, J.; Dijkgraaf, M.; Kruis, M.; Van Dasselaar, N.; Van der Vegt, M. Iontophoretic administration of S (+)-ketamine in patients with intractable central pain: A placebo-controlled trial. Pain 2005, 118, $224-231$. [CrossRef]

12. Wood, D.M.; Davies, S.; Puchnarewicz, M.; Johnston, A.; Dargan, P.I. Acute toxicity associated with the recreational use of the ketamine derivative methoxetamine. Eur. J. Clin. Pharmacol. 2012, 68, 853-856. [CrossRef] [PubMed]

13. Gein, V.; Levandovskaya, E.; Nosova, N.; Antonova, N.; Voronina, E.; Vakhrin, M.; Krivenko, A. Synthesis and antibacterial activity of $N, N^{\prime}$-diaryl-2-aryl-6-hydroxy-6-methyl-4-oxocyclohexane-1,3-dicarboxamides. Pharm. Chem. J. 2007, 41, 643-645. [CrossRef]

14. Holland, K.; Naritoku, D.; McKeon, A.; Ferrendelli, J.; Covey, D. Convulsant and anticonvulsant cyclopentanones and cyclohexanones. Mol. Pharmacol. 1990, 37, 98-103. [PubMed]

15. Liu, L.; Liu, S.; Chen, X.; Guo, L.; Che, Y. Pestalofones A-E, bioactive cyclohexanone derivatives from the plant endophytic fungus Pestalotiopsis fici. Biorg. Med. Chem. 2009, 17, 606-613. [CrossRef]

16. Adepu, R.; Rambabu, D.; Prasad, B.; Meda, C.L.T.; Kandale, A.; Krishna, G.R.; Reddy, C.M.; Chennuru, L.N.; Parsa, K.V.; Pal, M. Novel thieno [2,3-d] pyrimidines: Their design, synthesis, crystal structure analysis and pharmacological evaluation. Org. Biomol. Chem. 2012, 10, 5554-5569. [CrossRef]

17. Kumar, T.B.; Dhananjaya, G.; Sumanth, C.; Vaishaly, S.; Botre, G.; Rao, M.S.; Sekhar, K.C.; Kumarc, K.S.; Pal, M. Indion 860 catalyzed cascade reaction: A greener approach to functionalized cyclohexanones and their novel analogues3. RSC Adv. 2013, 3, 2207-2210. [CrossRef]

18. Yang, H.; Rudge, D.G.; Koos, J.D.; Vaidialingam, B.; Yang, H.J.; Pavletich, N.P. mTOR kinase structure, mechanism and regulation. Nature 2013, 497, 217. [CrossRef]

19. Stamos, J.; Sliwkowski, M.X.; Eigenbrot, C. Structure of the epidermal growth factor receptor kinase domain alone and in complex with a 4-anilinoquinazoline inhibitor. J. Biol. Chem. 2002, 277, 46265-46272. [CrossRef]

20. Aronov, A.M.; Baker, C.; Bemis, G.W.; Cao, J.; Chen, G.; Ford, P.J.; Germann, U.A.; Green, J.; Hale, M.R.; Jacobs, M. Flipped out: Structure-guided design of selective pyrazolylpyrrole ERK inhibitors. J. Med. Chem. 2007, 50, 1280-1287. [CrossRef] 
21. Feoktistova, M.; Geserick, P.; Leverkus, M. Crystal violet assay for determining viability of cultured cells. Cold Spring Harbor Protocols 2016, 2016. [CrossRef]

22. Mosmann, T. Rapid colorimetric assay for cellular growth and survival: Application to proliferation and cytotoxicity assays. J. Immunol. Methods 1983, 65, 55-63. [CrossRef]

23. Slater, T.; Sawyer, B.; Sträuli, U. Studies on succinate-tetrazolium reductase systems: III. Points of coupling of four different tetrazolium salts III. Points of coupling of four different tetrazolium salts. Biochim. Biophys. Acta 1963, 77, 383-393. [CrossRef]

24. Muchmore, S.W.; Souers, A.J.; Akritopoulou-Zanze, I. The use of three-dimensional shape and electrostatic similarity searching in the identification of a melanin-concentrating hormone receptor 1 antagonist. Chem. Biol. Drug Des. 2006, 67, 174-176. [CrossRef] [PubMed]

25. Abdellatif, K.R.; Fadaly, W.A.; Kamel, G.M.; Elshaier, Y.A.; El-Magd, M.A. Design, synthesis, modeling studies and biological evaluation of thiazolidine derivatives containing pyrazole core as potential anti-diabetic PPAR- $\gamma$ agonists and anti-inflammatory COX-2 selective inhibitors. Bioorg. Chem. 2019, 82, 86-99. [CrossRef]

26. Likhitwitayawuid, K.; Angerhofer, C.K.; Chai, H.; Pezzuto, J.M.; Cordell, G.A.; Ruangrungsi, N. Cytotoxic and antimalarial alkaloids from the tubers of Stephania pierrei. J. Nat. Prod. 1993, 56, 1468-1478. [CrossRef] [PubMed]

27. Barakat, A.; Islam, M.S.; Ghawas, H.M.; Al-Majid, A.M.; El-Senduny, F.F.; Badria, F.A.; Elshaier, Y.A.M.; Ghabbour, H.A. Substituted spirooxindole derivatives as potent anticancer agents through inhibition of phosphodiesterase 1. RSC Adv. 2018, 8, 14335-14346. [CrossRef]

28. Kazeem, M.I.; Ogunbiyi, J.V.; Ashafa, A.O. In vitro studies of the inhibition of a-amylase and $\alpha$-glucosidase by leaf extracts of Picralimanitida (Stapf). Trop. J. Pharm. Res. 2013, 12, 719-725.

29. Fast Rigid Exhaustive Docking (FRED) Receptor, version 2.2.5; Open Eye Scientific Software: Santa Fe, NM, USA, 2019; Available online: http:/ / www.eyesopen.com (accessed on 13 March 2019).

30. OMEGA, version 2.5.1.4; Open Eye Scientific Software: Santa Fe, NM, USA, 2019; Available online: http:/ / www.eyesopen.com (accessed on 13 March 2019).

31. VIDA, version 4.1.2; Open Eye Scientific Software: Santa Fe, NM, USA, 2019; Available online: http:/ / www.eyesopen.com (accessed on 13 March 2019).

Sample Availability: Samples of the compounds 3a-m are available from the authors.

(C) 2019 by the authors. Licensee MDPI, Basel, Switzerland. This article is an open access article distributed under the terms and conditions of the Creative Commons Attribution (CC BY) license (http://creativecommons.org/licenses/by/4.0/). 